\title{
The Simulation Study on Deep-sea Microbes Sampling of Holding the In-Situ Pressure Based on the Pressure Compensation with 316L Stainless Steel Accumulator
}

\author{
Song Wenjie ${ }^{1, a}$, Liu Peng ${ }^{1, b^{*}}$, Jiang Qinglin ${ }^{1, c}$ and Li Xinjuan ${ }^{1, d}$ \\ ${ }^{1}$ Shandong Provincial Key Laboratory of Ocean Environment Monitoring Technology, Institute of \\ Oceanographic Instrumentation, Shandong Academy of Sciences, Qingdao 266001, China

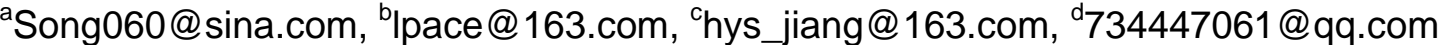

Keywords: Accumulator, 316L Stainless Steel, Pressure compensation, Deep-sea microbes, Simulation study

\begin{abstract}
This paper proposes a physical model of pressure compensation with accumulator based on the simulation research. The best accumulator can be chosen quickly and reasonably for different sampling systems. The mathematical model about the final pressure of the accumulator is established. The accumulator models with different nominal volumes are analyzed in variety pre-charged pressures. The simulation results of the system show that: When the nominal capacity of accumulator is $1.6 \mathrm{~L}$ and the pre-charged pressure is $25 \mathrm{MPa}$, the system can meet the design requirements of $310 \mathrm{~mL}$ sampling vessel, and the structure of the sampling system can reach the optimal state.
\end{abstract}

\section{Introduction}

The deep-sea microbes have strict requirements on the living environment [1]. Because the ordinary sampler volume is limited, it is difficult to maintain the in-situ pressure [2]. Due to the shortage of ordinary sampler, a new sampling technique with function of concentrated sampling and holding the in-situ pressure is needed. It will increase the number of microbes and keep characteristic of the microbes in in-situ pressure [3].

The United States and other developed countries have carried out the study on pressure compensation technique, and the microbe of depth $4000 \mathrm{~m}$ has been obtained $[4,5]$. Our country is lagging behind other countries. Under the support of the National 863 program, the device for holding pressure was developed. The device is limited to sampling in holding pressure, which is not suitable for a large number of sampling for microbes [6, 7]. Through investigation we found that there are still many deficiencies existed in current sampling technology.

Accumulator is one of the most important components in the holding pressure sampling system. In this paper, a simulation study method for accumulator is used to study the actual opening pressure, the relationship between pre-charged pressure and the ability of pressure-retaining, the relationship between the pre-charged pressure and the minimum working volume, the relationship between nominal volume and the ability of pressure-retaining, which provides a theoretical guidance for the practical design.

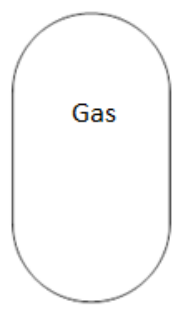

(1)

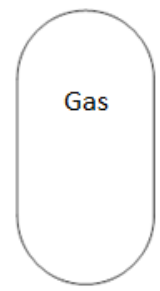

(2)

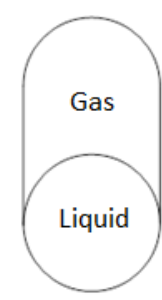

(3)

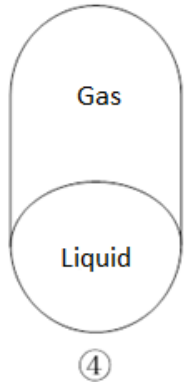

(4)

Fig. 1 The changing process of gas pressure in accumulator. 


\section{Study on the Final Pressure Model of Sample}

Set that the accumulator is cylindrical. Where, $r_{x}$ is the inner diameter, $R_{x}$ is the outer diameter, $h_{x}$ is the height, $E_{x}$ and $\mu_{x}$ is Elastic Modulus and Poisson's Ratio. Set that the sampling vessel is cylindrical. $r_{t}$ is the inner diameter, $R_{t}$ is the outer diameter, height is $h_{t}$, Elastic Modulus and Poisson's Ratio is $E_{t}$ and $\mu_{t}$. The process of decentralization and recovery is slow, and the water in the surrounding is cold. It can be considered that the gas in the whole sampling process is in isothermal variation.

From the state (1) to the state (2), the gas in the accumulator is compressed, which leads to the opening pressure of the accumulator increased. The equation of the gas state is as follows:

$$
V_{x 1}=\pi\left(r_{x}+\Delta r_{x}\right)^{2}\left(h_{x}+\Delta h_{x}\right)
$$

$\Delta r_{\mathrm{x} 1}$ and $\Delta h_{\mathrm{x} 1}$ are the radial displacement and the axial displacement of the cylinder of accumulator. Because $\Delta r_{\mathrm{x} 1}$ and $\Delta h_{\mathrm{x} 1}$ are very small, the quadratic term can be ignored.

Let be: $k_{1}=2 \pi r_{x} h_{x}, k_{2}=\pi r_{x}^{2}, k_{3}=\frac{\left(1-2 \mu_{x}\right) r_{x}^{3}}{E_{x}\left(R_{x}^{2}-r_{x}^{2}\right)}, k_{4}=\frac{\left(1+\mu_{x}\right) R_{x}^{2} r_{x}}{E_{x}\left(R_{x}^{2}-r_{x}^{2}\right)}, k_{5}=\frac{\left(1-2 \mu_{x}\right) h_{x} r_{x}^{2}}{E_{x}\left(R_{x}^{2}-r_{x}^{2}\right)}$

The radial displacement and the axial displacement of the accumulator are as follows:

$$
\begin{aligned}
& \Delta r_{x 1}=p_{x 1}\left(k_{3}+k_{4}\right) \\
& \Delta h_{x 1}=p_{x 1} k_{5}
\end{aligned}
$$

By formula (1) - (3), we can get the opening pressure of accumulator is as follows:

$$
p_{x 2}=p_{x 1}\left(1+\frac{k_{1} p_{x 1}\left(k_{3}+k_{4}\right)}{k_{2} h_{x}}+\frac{p_{x 1} k_{5}}{h_{x}}\right)
$$

From the state (3) to the state (4), the sampling vessel is in the process of recovery. The pressure of the sampling vessel will be compensated at the same time:

$$
V_{x 4}=V_{x 3}+\Delta V_{x 4}+\Delta V_{t 4}
$$

$\triangle V_{\mathrm{x} 4}$ is the volume deformation of the accumulator, $\Delta V_{\mathrm{t} 4}$ is the volume deformation of the sampling vessel.

Let be: $k_{6}=2 \pi r_{t} h_{t}, k_{7}=\pi r_{t}^{2}, k_{8}=\frac{\left(1-2 \mu_{t}\right) r_{t}^{3}}{E_{t}\left(R_{t}^{2}-r_{t}^{2}\right)}, k_{9}=\frac{\left(1+\mu_{t}\right) R_{t}^{2} r_{t}}{E_{t}\left(R_{x}^{2}-r_{t}^{2}\right)}, k_{10}=\frac{\left(1-2 \mu_{t}\right) h_{t} r_{t}^{2}}{E_{t}\left(R_{t}^{2}-r_{t}^{2}\right)}$.

Volume deformation of the sampling vessel is:

$$
\Delta V_{t 4}=p_{t 3}\left(k_{6}\left(k_{8}+k_{9}\right)+k_{7} k_{10}\right)
$$

Volume deformation of the accumulator is:

$$
\Delta V_{x 4}=p_{x 3}\left(k_{1}\left(k_{3}+k_{4}\right)+k_{2} k_{5}\right)
$$

By formula (7) - (14), we can get the final gas pressure of the accumulator:

$$
p_{x 4}=\frac{p_{x 3} V_{x 3}}{V_{x 3}+p_{t 3}\left(k_{6}\left(k_{8}+k_{9}\right)+k_{7} k_{10}\right)+p_{x 3}\left(k_{1}\left(k_{3}+k_{4}\right)+k_{2} k_{5}\right)}
$$

The accumulator and the sampling vessel must meet the following conditions: (1) After the recovery of the sampling vessel, the gas pressure of the accumulator is greater than the pre-charged pressure; (2) The volume of gas in the accumulator is smaller than the pre-charged gas volume; (3) The sample pressure in the sampling vessel is equal to the gas pressure in the accumulator. 


\section{The Simulation Study on the Pressure-Retaining Capacity of Accumulator}

The MATLAB simulation program was used to study the opening pressure of the accumulator, the pre-charged pressure of accumulator, the volume of accumulator, the relationship between the working volume and the holding pressure effect of the accumulator. The parameters used in the simulation research are shown in Table 1.

Table 1. Simulation parameters.

\begin{tabular}{llc}
\hline \multicolumn{1}{c}{ parameters } & \multicolumn{1}{c}{ Value } & unit \\
\hline Elastic Modulus of accumulator: $E_{x}$ & $200 \times 10^{9}$ & $\mathrm{~Pa}$ \\
Poisson's Ratio of accumulator: $\mu_{x}$ & 0.3 & - \\
The inner diameter of accumulator: $r_{x}$ & $0.03 / 0.04 / 0.05 / 0.06 / 0.07$ & $m$ \\
The outer diameter of accumulator: $R_{x}$ & $0.05 / 0.065 / 0.080 / 0.095 / 0.11$ & $m$ \\
The height of accumulator: $h_{x}$ & 0.2 & $m$ \\
Elastic Modulus of sampling vessel: $E_{t}$ & $190 \times 10^{9}$ & $\mathrm{~Pa}$ \\
Poisson's Ratio of sampling vessel: $\mu_{t}$ & 0.3 & - \\
The inner diameter of sampling vessel: $r_{t}$ & 0.03 & $m$ \\
The outer diameter of sampling vessel: $R_{t}$ & 0.05 & $m$ \\
The height of sampling vessel: $h_{t}$ & 0.11 & $m$ \\
Pressure of sampling point: $p_{x 3}$ & $60 \times 10^{6}$ & $\mathrm{~Pa}$ \\
Gas pressure of pre-charged: $p_{x 1}$ & $(20 \sim 50) \times 10^{6}$ & $\mathrm{~Pa}$ \\
\hline
\end{tabular}

The Simulation Analysis of the Actual Opening Pressure of Accumulator. Fig. 2 indicates the relationship between the pre-charged pressure and the increase quantity of the actual opening pressure of accumulator in various nominal volumes. With the pre-charged pressure increasing, the actual opening pressure increases faster and the trend of growth is gradually enhanced. Compared with the maximum nominal volume of accumulator, the pre-charged pressure of the minimum nominal volume accumulator is in the range of $20 \mathrm{MPa}$ to $50 \mathrm{MPa}$. The increase quantity of $\Delta p$ is only $0-5000 \mathrm{~Pa}$, but the wall thickness of the accumulator increases from $20 \mathrm{~mm}$ to $40 \mathrm{~mm}$. There is a large increase on the weight of the accumulator.

The Relationship between the Pre-Charged Pressure of Accumulator and the Final Pressure of Sample. Fig. 3 reflects the relationship between the pre-charged pressure of accumulator and the final pressure of the sampling vessel. When the pre-charged pressure of accumulator increases, the final pressure of the sampling vessel increases slowly, but the increasing trend is gradually weakened.

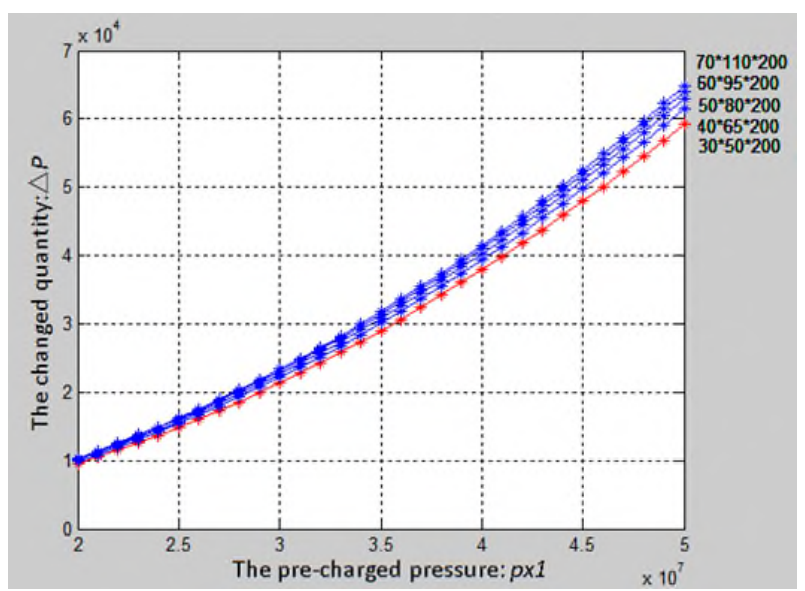

Fig. 2 The relationship between the pre-charged pressure and the increase quantity of the actual opening pressure of accumulator in various nominal volumes.

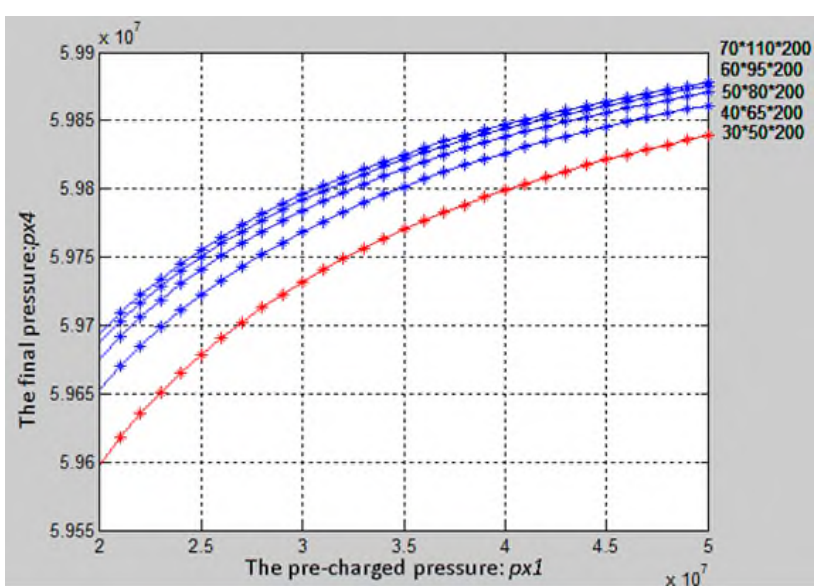

Fig. 3 The relationship between the pre-charged pressure and the final pressure of the sampling vessel. 
Under the same pre-charged pressure, the final pressure of the sampling vessel increases with the increases of the nominal volume of the accumulator, but the increasing trend is gradually reduced. The wall thickness of the accumulator increases greatly. On the contrary, the pressure of accumulator doesn't increase obviously.

The Relationship between the Pre-Charged Pressure and the Minimum Working Volume of the Accumulator. Fig. 4 is the relationship between the pre-charged pressure and the volume of peltry. When the pre-charged pressure of accumulator is low, the gas in the peltry is massively compressed. Under the same pre-charged pressure, the curve's slope increases gradually with the increase of the nominal volume, this shows that the accumulator with a larger volume is easily affected by the pre-charged pressure. The accumulator is easier to go to the critical point of $25 \%$.

In the process of pressure compensation, the volume of peltry doesn't increase obviously. When the nominal volume of peltry becomes bigger, the volume of peltry changes faster. It shows that the larger the nominal volume of accumulator is, the more sensitive the accumulator to the change of pressure, the more unstable the performance of the accumulator.

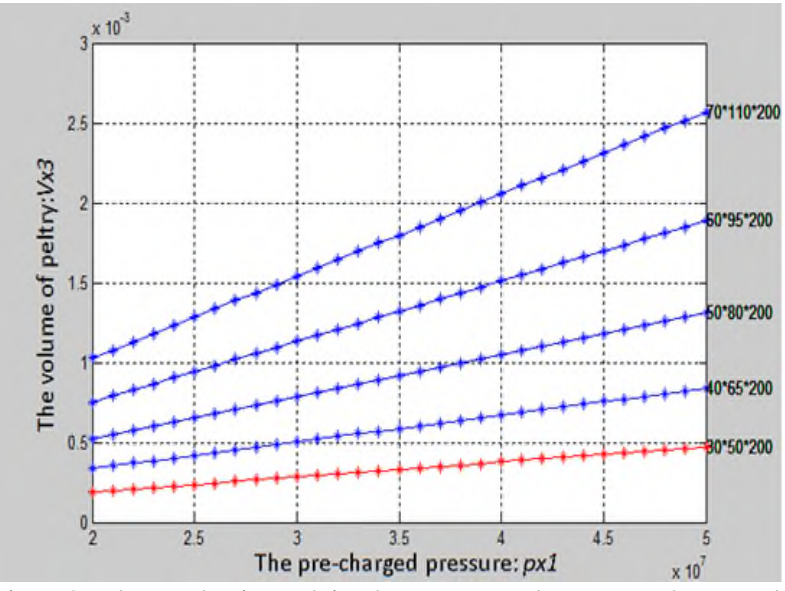

Fig. 4 The relationship between the pre-charged pressure and the volume of peltry.

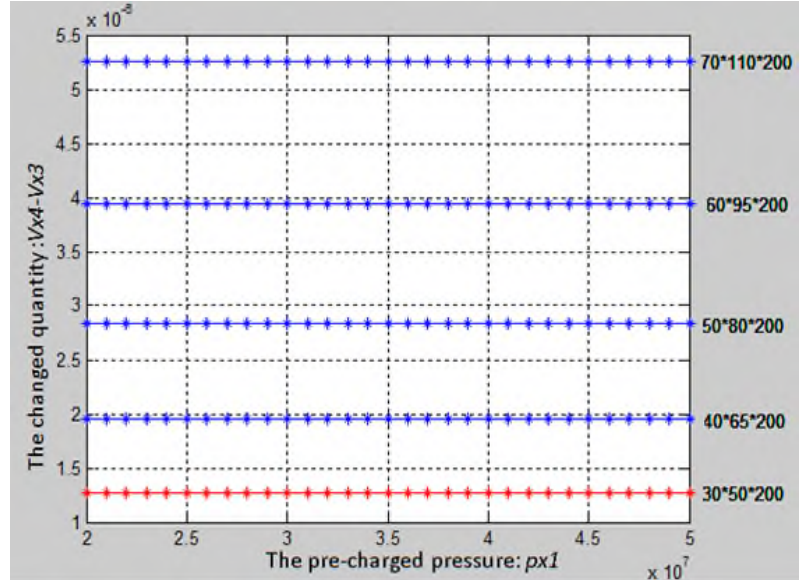

Fig. 5 Changes in the volume of peltry.

The Relationship between Nominal Volume and Final Pressure of Accumulator. When the pre-charged pressure increases from $20 \mathrm{MPa}$ to $50 \mathrm{MPa}$, the relationship between nominal volume and the final pressure of accumulator is shown in Fig. 6. With the increase of the pre-charged pressure, the sampling points become more and more intensive, which shows that the final pressure doesn't increase synchronously with the pre-charged pressure.

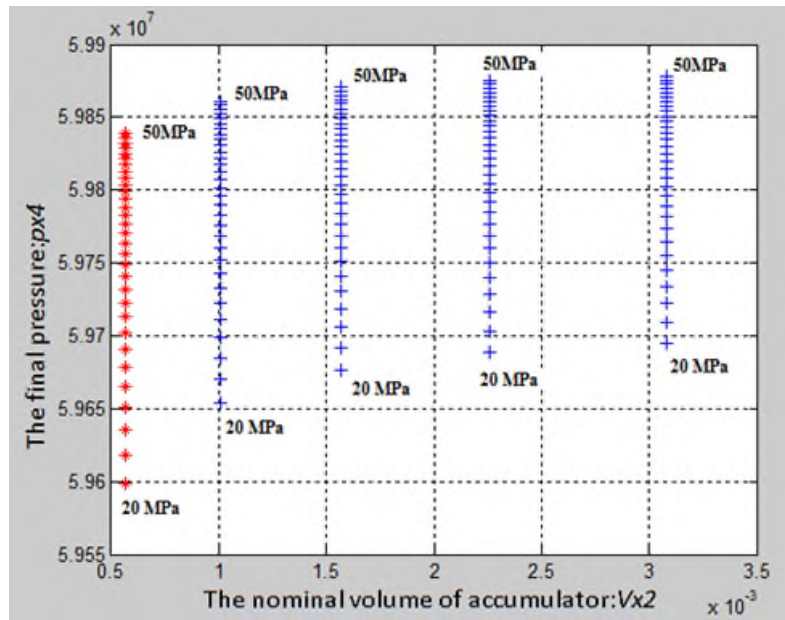

Fig. 6 The relationship between nominal volume and final pressure of accumulator.

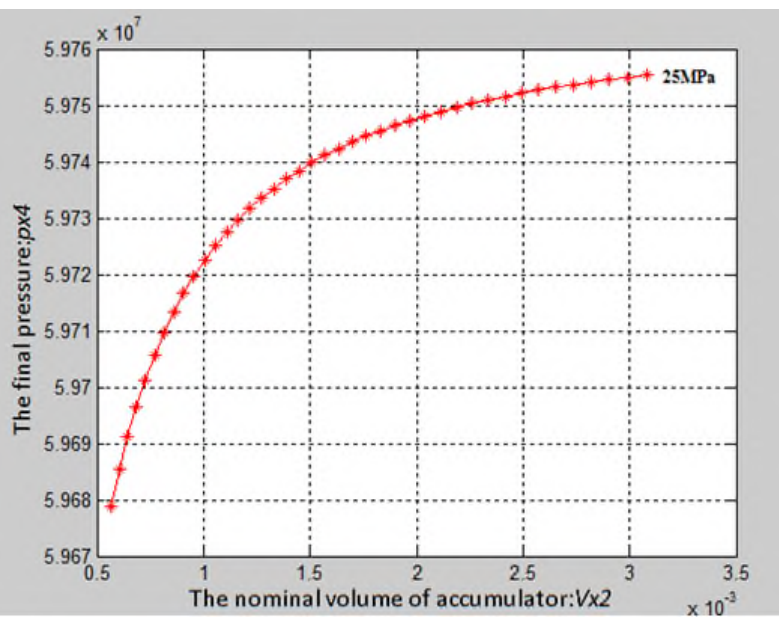

Fig. 7 The relationship between nominal volume and final pressure when the pre-charged pressure is $25 \mathrm{MPa}$. 
With the increase of nominal volume of accumulator, the final pressure also increase, but the increase maximum is limited. Take the pre-charged pressure of $25 \mathrm{MPa}$ as an example, as shown in Fig. 7. When the nominal volume of the accumulator is less than $2 \mathrm{~L}$, increasing the nominal volume of the accumulator can increase the final pressure of the accumulator significantly. When the nominal volume of accumulator is greater than $2 \mathrm{~L}$, the final pressure of the accumulator is not obvious. The sampling vessel of this paper is $310 \mathrm{~mL}$. When the nominal volume of accumulator is below $2 \mathrm{~L}$, the highest utilization rate can be obtained. The accumulator with nominal volume 1.6L is selected as the pressure compensator, which can make the pre-charged pressure up to $25 \mathrm{MPa}$.

\section{Experiment}

Materials. The accumulator with 316L Stainless Steel is cylindrical. Where, $r_{\mathrm{x}}$ is the inner diameter, $R_{\mathrm{x}}$ is the outer diameter, $h_{\mathrm{x}}$ is the height, $E_{\mathrm{x}}$ and $\mu_{\mathrm{x}}$ is Elastic Modulus and Poisson's Ratio. The sampling vessel with 316L Stainless Steel is cylindrical. $r_{\mathrm{t}}$ is the inner diameter, $R_{\mathrm{t}}$ is the outer diameter, height is $h_{\mathrm{t}}$, Elastic Modulus and Poisson's Ratio is $E_{\mathrm{t}}$ and $\mu_{\mathrm{t}}$. and the all parameter values were shown in Table 1.

Methods .The mathematical model about the final pressure of the accumulator is established. The accumulator models with different nominal volumes are analyzed in variety pre-charged pressures and the results were shown in Fig. 2-7.

\section{Summary and Conclusion}

1. On the condition of ensuring the opening pressure stability, the wall thickness of the accumulator increases fast along with the increase of the nominal volume and pre-charged pressure. The weight of the accumulator increases in a great extent.

2. When the pre-charged pressure of accumulator increases, the final pressure of the sampling vessel increases slowly and the increasing trend is gradually weakened. It is difficult to obtain a large pre-charged pressure by using ordinary filling devices. Therefore, there is no need to use large accumulator with high pre-charged pressure.

3. In the same pre-charged pressure, the increase of the final pressure of the sampling vessel is not along with the increase of the nominal volume. When the nominal volume of the accumulator is selected appropriately, the pressure requirements of system can be satisfied and the service life of the accumulator can be prolonged.

4. Through the study of this paper, when the nominal volume of the sampling vessel is set as $310 \mathrm{~mL}$, the normal volume of accumulator is $1.6 \mathrm{~L}$ and pre-charged pressure of the pressure compensator is $25 \mathrm{MPa}$, the sampling system will get a best performance.

\section{Acknowledgement}

The result of this research is supported by the Key Research and Development Program of Shandong Province (NO.2015GSF115003), Qingdao City, Shinan District Science and Technology Development Project Funds(NO.2014-14-010-SW), The Youth Fund Project of Shandong Academy of Sciences (NO.2014QN040), Qingdao Application Foundation Research Project (NO.15-9-1-84-JCH).

\section{References}

[1] D. E. Helbling, M. Ackermann, K. Fenner, etc. The activity level of a microbial community function can be predicted from its metatranscriptome. ISME J. 6 (2012) 902-904.

[2] J. Feike, K. Jürgens, J. T. Hollibaugh, etc. Measuring unbiased metatranscriptomics in suboxic waters of the central Baltic Sea using a new in situ fixation system. ISME J. 6 (2012) 461-470.

[3] J. K. B. Bishop, P. J. Lam, T. J. Wood. Getting good particles: accurate sampling of particles by 
large volume in-situ filtration. Limnol. Oceanogr. Meth. 10 (2012) 681-710.

[4] K. E. Wommack, S. J. Williamson, A. Sundbergh. An instrument for collecting discrete large-volume water samples suitable for ecological studies of microorganisms. Deep. Sea. Res. Part I, 51 (2004) 1781-1792.

[5] A. Bianchi, J. Garcin, O. Tholosan. A high-pressure serial sampler to measure microbial activity in the deep sea. Deep Sea Res. Part I, 46 (1999) 2129-2142.

[6] W. Li. The critical technology of deep-sea hydrothermal fluid holding pressure sampler. Zhejiang University, doctoral dissertation, 2007.

[7] L. An, Y. Cheng, H. W. Qin, etc. Parametric influence analyses for the pressure-holding characteristics of air-cushion fidelity canister. Ocean Eng. 32(3) (2014) 110-115. 